\title{
Penfigoide ampollar inducido por gliptinas
}

\section{Bullous pemphigoid induced by gliptins}

\author{
Marianela Geloso ${ }^{1}$, María Emilia Candiz², Olga Forero ${ }^{2}$, Liliana Olivares ${ }^{3}$ y Esteban Maronna ${ }^{4}$
}

\section{RESUMEN}

Las gliptinas son hipoglucemiantes utilizados para el tratamiento de la diabetes mellitus tipo 2, como monoterapia o junto con la metformina. Desde su incorporación, en 2006, se han comunicado múltiples casos que evidencian su asociación con el penfigoide am- pollar. Se presenta el caso de un paciente de 83 años con diagnóstico de penfigoide ampollar inducido por vildagliptina.

Palabras clave: gliptinas, penfigoide ampollar, vildagliptina.

Dermatol. Argent. 2020, 26 (4): 167-169

\section{ABSTRACT}

Gliptins are hypoglycemic drugs used to treat type 2 diabetes mellitus, as monotherapy or together with metformin. They were incorporated in 2006 and since then there have been multiple reported cases that demonstrate their association with bullous pemphigoid. We report a case of an 83-year-old patient with a diagnosis of vildagliptin-induced bullous pemphigoid.

Key words: gliptins, bullous pemphigoid, vildagliptin.

Dermatol. Argent. 2020, 26 (4): 167-169
${ }^{1}$ Médica Concurrente, Segundo Año de Dermatología

${ }^{2}$ Médica de Planta, Especialista en Dermatología

3 Jefa, Unidad de Dermatología

${ }^{4}$ Dermatopatólogo

Unidad de Dermatología, Hospital Dr. Francisco J. Muñiz, Ciudad Autónoma de Buenos Aires, Argentina
Contacto del autor: Marianela Geloso E-mail: marugeloso@gmail.com

Fecha de trabajo recibido: $15 / 5 / 2020$

Fecha de trabajo aceptado: 21/11/2020

Conflicto de interés: los autores declaran que no existe conflicto de interés.

\section{CASO CLÍNICO}

Un paciente de 83 ańos, con antecedentes de hipertensión arterial, diabetes mellitus tipo 2 e insuficiencia renal, consultó por padecer una dermatosis generalizada y pruriginosa, de 2 meses de evolución. En el interrogatorio refirió la incorporación de la vildagliptina (hipoglucemiante oral) un mes antes del inicio del cuadro.

En el examen físico se evidenciaron, en el tronco y las extremidades, grandes placas eritematoedematosas, que alternaban con erosiones de fondo rojizo y ampollas tensas de diferentes tamaños, con contenido seroso y se- rohemático (Foto 1). Algunas ampollas asentaban sobre piel sana y otras, sobre piel eritematoedematosa (Foto 2).

Se solicitaron los siguientes exámenes complementarios: rutina de laboratorio con hemograma, hepatograma y función renal: sin particularidades. Citodiagnóstico de Tzanck: inflamación inespecífica. Biopsia de la piel de una ampolla en el brazo izquierdo para histopatología: despegamiento ampollar subepidérmico (Foto 3) y extensos sectores de necrosis epidérmica en los estratos medios y basales. En la dermis, infiltrados inflamatorios constituidos por neutrófilos y eosinófilos (Foto 4). Inmunofluo- 


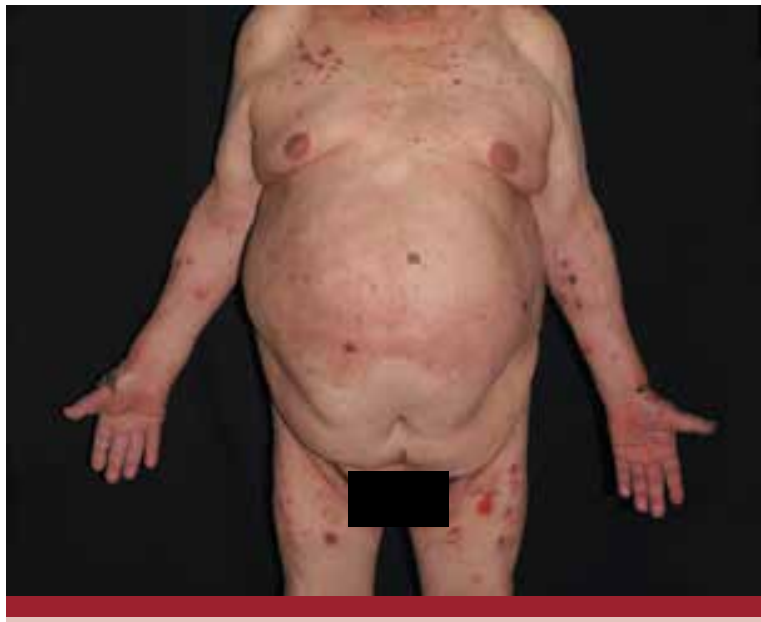

FOTO 1: Placas eritematoedematosas, erosiones y ampollas en el tronco y las extremidades.

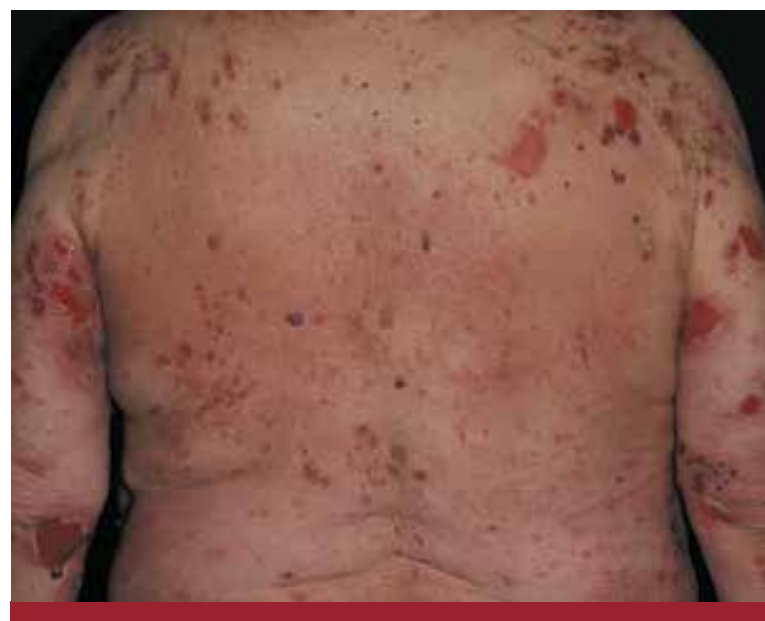

FОTO 2: Ampollas tensas y erosiones, algunas sobre piel sana y otras sobre una base eritematosa.

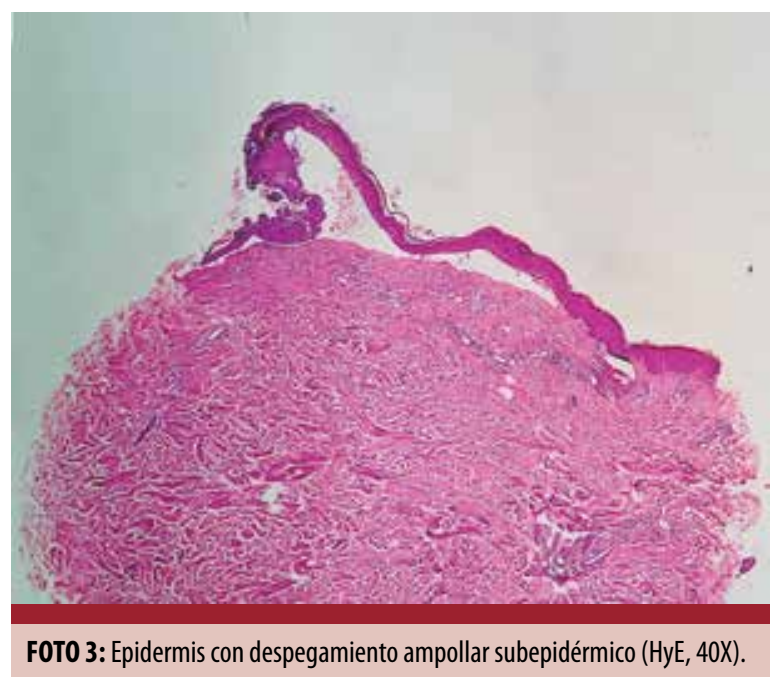

rescencia directa: depósitos de anti-IgG y anti-C3 con patrón lineal en la zona de la membrana basal. Técnica de salt-split. IgG en el costado epidérmico. Serologías por

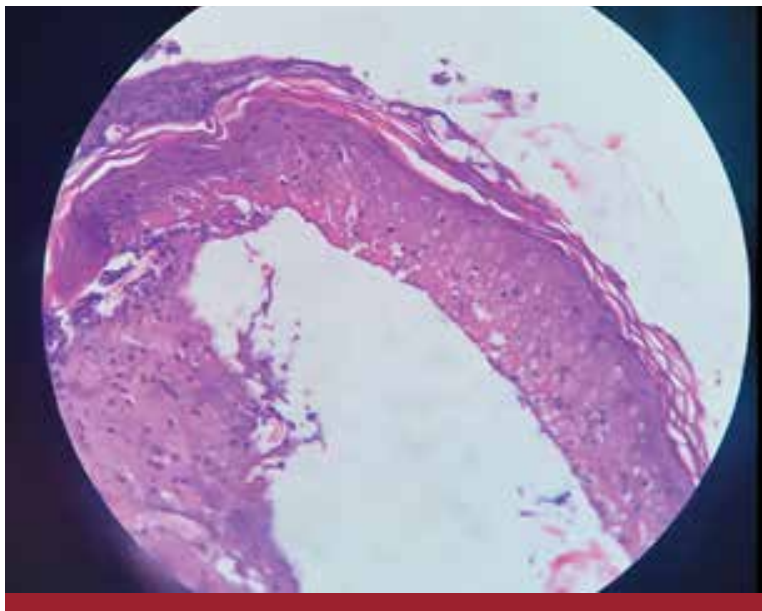

FOTO 4: Techo de una ampolla con extensa necrosis epidérmica que compromete todo el espesor del epitelio. En la dermis, infiltrados inflamatorios constituidos por neutrófilos y eosinófilos (HyE, 400X).

ELISA: positivas para BP 180 y BP 230. Se realizó la escala de Naranjo, que arrojó un resultado de 4 puntos (asociación posible).

Con estos resultados, se arribó al diagnóstico de penfigoide ampollar inducido por gliptinas (PA-IG).

Se suspendió la medicación y se realizó tratamiento con dexametasona $8 \mathrm{mg} / \mathrm{d}$ por vía intravenosa durante 15 días, con posterior descenso gradual, asociado a minociclina $200 \mathrm{mg} /$ día por vía oral. El paciente tuvo una buena evolución clínica sin nuevos despegamientos ampollares y discontinuó el seguimiento.

\section{COMENTARIOS}

El penfigoide ampollar (PA) es una de las enfermedades ampollares autoinmunes más frecuentes. Si bien en la mayoría de los pacientes no se identifica un agente causal, recientemente se lo relacionó con el uso de ciertos fármacos como desencadenantes ${ }^{1}$.

En los últimos años, surgió una fuerte evidencia sobre la asociación del PA y el uso de los inhibidores de la dipeptidil peptidasa-4 (IDPP-4), también llamados gliptinas. Estos medicamentos son hipoglucemiantes utilizados para el tratamiento de la diabetes mellitus 2, como monoterapia o junto con la metformina. Introducidas en 2006, en la actualidad existen cinco IDPP-4 globalmente disponibles: sitagliptina, vildagliptina, saxagliptina, linagliptina y alogliptina ${ }^{2}$.

Skandalis et ál. fueron los primeros en describir esta asociación en 2011. Publicaron una serie con 5 pacientes que desarrollaron PA-IG (4 vildagliptina, 1 sitagliptina), con un período de latencia de entre 2 y 13 meses desde la incorporación del fármaco. La clínica y la histopatología fueron indistinguibles de las del PA idiopático y, en todos ellos, la enfermedad se controló después de retirada la medicación sospechosa ${ }^{3}$. 
Desde entonces, se ha incrementado la cantidad de comunicaciones, incluso, con estudios aleatorizados y controlados $^{4-8}$. Todos ellos afirman que existe una asociación estadísticamente significativa entre el consumo de gliptinas (excepto la alogliptina) y la aparición de $\mathrm{PA}$. El riesgo relativo varía según la gliptina analizada y las diferentes investigaciones. La vildagliptina es la que se asoció con mayor frecuencia, como en el paciente del caso analizado, y sería independiente del consumo concomitante o no de metformina ${ }^{4-8}$.

El mecanismo exacto por el cual las gliptinas desencadenan el PA no está definido. Se demostró la expresión de la enzima dipeptidil peptidasa-4 en la piel como receptores de superficie en queratinocitos y células $\mathrm{T}$. Su inhibición provoca la activación de eosinófilos por un mecanismo mediado por CCL11/eotaxina, lo que contribuye a la formación de ampollas ${ }^{3,4}$.

El período de latencia es muy variable. La base de datos de farmacovigilancia europea y francesa EudraVigilance identificó, hasta 2015, 113 casos de PA asociado a la vildagliptina, con predominio en los hombres y los mayores de 70 años, y con un período de latencia desde la incorporación del fármaco hasta el inicio de la dermatosis de 6 a 19 meses 4 .

En general, no hay diferencias clínicas con el PA idiopático, como en el caso analizado. Sin embargo, Izu-

\section{BIBLIOGRAFÍA}

1. Stavropoulos PG, Soura E, Antoniou C. Drug-induced pemphigoid: a review of the literature. J Eur Acad Dermatol Venereol 2014;28:1133-1140.

2. Aylwin C. Nuevos fármacos en diabetes mellitus. Rev Med Clin Condes 2016;27:235-256.

3. Skandalis K, Spirova M, Gaitanis G, Tsartsarakis A, et ál. Druginduced bullous pemphigoid in diabetes mellitus patients receiving dipeptidyl peptidase-IV inhibitors plus metformin. J Eur Acad Dermat Venereol 2012;26:249-253.

4. García M, Aranburu M, Palacios-Zabalza I, Lertxundi U, et ál. Dipeptidyl peptidase-IV inhibitors induced bullous pemphigoid: a case report and analysis of cases reported in the European pharmacovigilance database. J Clin Pharm Ther 2016;41:368-370.

5. Reolid A, Muñoz-Aceituno E, Rodríguez-Jiménez P, GonzálezRojano $\mathrm{E}$, et ál. Bullous pemphigoid associated with dipeptiyl peptidase-4 inhibitors. A case series and analysis of cases reported in the Spanish pharmacovigilance database. Int $J$ Dermatol 2020;59:197-206. mi et ál. describieron una fuerte asociación del PA-IG con un fenotipo no inflamatorio, caracterizado por ampollas pequeñas con eritema leve o sin él y extensión limitada de la dermatosis. Estos autores vincularon este fenotipo a un perfil de autoanticuerpos (Ac) que reconocen epítopos diferentes del dominio NC16A del BP180 (principal blanco antigénico del PA clásico) ${ }^{9}$. Se destaca que los kits serológicos de ELISA disponibles en la Argentina solo permiten detectar Ac contra el dominio NC16A y, por lo tanto, en el caso del PA no inflamatorio estas pruebas no serían de utilidad.

Con respecto al compromiso mucoso, a diferencia de otros autores, Kridin et ál. observaron una mayor frecuencia de afectación en los pacientes con PA-IG en comparación con la variedad idiopática ${ }^{8}$.

En la histopatología del paciente del caso se destaca la necrosis de queratinocitos en la epidermis, hallazgo comunicado en otras entidades ampollares autoinmunes desencadenadas por fármacos, como la dermatosis por IgA lineal de ese origen ${ }^{10}$.

La mayoría de los trabajos publicados muestran que con la suspensión de la medicación se logra la remisión parcial o total de la dermatosis, en muchas ocasiones sin necesidad de iniciar tratamientos sistémicos ${ }^{4-6}$.

El interés de esta publicación es destacar la creciente asociación de las gliptinas como desencadenantes de PA.

6. García-Díez I, Ivars-Lleó M, López-Aventín D, Ishii N, et ál. Bullous pemphigoid induced by dipeptidyl peptidase-4 inhibitors. Eight cases with clinical and immunological characterization. Int J Dermatol 2018;57:810-816.

7. Phan K, Charlton O, Smith S. Dipeptidyl peptidase-4 inhibitors and bullous pemphigoid: A systematic review and adjusted meta-analysis. Australas J Dermatol 2020;61:e15-e21.

8. Kridin K, Bergman R. Association of Bullous Pemphigoid With Dipeptidyl-Peptidase 4 Inhibitors in Patients With Diabetes. JAMA Dermatol 2018;154:1152-1158.

9. Izumi K, Nishie W, Mai Y, Wada M, et ál. Autoantibody Profile Differentiates between Inflammatory and Noninflammatory Bullous Pemphigoid. J Invest Dermat 2016;136:2201-2210.

10. Rodríguez L, Forero O, Olivares L, Candiz ME, et ál. Dermatosis por IgA lineal vinculada a fármacos. Dermatol Argent 2017;23:42-45. 